\title{
Inconsistent Condom Use among Public Primary Care Patients with Tuberculosis in South Africa
}

\author{
Gladys Matseke, ${ }^{1}$ Karl Peltzer, ${ }^{1,2}$ Julia Louw, ${ }^{1}$ Pamela Naidoo, ${ }^{1,3}$ \\ Gugu Mchunu, ${ }^{1}$ and Bomkazi Tutshana ${ }^{1}$ \\ ${ }^{1}$ HIV/AIDS, STI, and TB (HAST) Research Programme, Human Sciences Research Council, Pretoria, Private Bag X41, \\ Pretoria 0001, South Africa \\ ${ }^{2}$ Department of Psychology, University of the Free State, Bloemfontein, South Africa \\ ${ }^{3}$ Department of Psychology, University of the Western Cape, Cape Town, South Africa \\ Correspondence should be addressed to Karl Peltzer, kpeltzer@hsrc.ac.za
}

Received 11 March 2012; Accepted 3 May 2012

Academic Editors: J. Arbiza, A. Arminio Monforte, and P. Chou

Copyright ( $\odot 2012$ Gladys Matseke et al. This is an open access article distributed under the Creative Commons Attribution License, which permits unrestricted use, distribution, and reproduction in any medium, provided the original work is properly cited.

\begin{abstract}
The high rate of HIV infections among tuberculosis (TB) patients in South Africa calls for urgent HIV reduction interventions in this subpopulation. While correct and consistent condom use is one of the effective means of HIV prevention among sexually active people, there is insufficient research on condom use among TB patients in South Africa. The aim of this paper was to determine the prevalence of inconsistent condom use among public primary care TB patients and its associated factors using a sample of 4900 TB patients from a cross-sectional survey in three health districts in South Africa. Results indicated that when asked about their consistency of condom use in the past 3 months, $63.5 \%$ of the participants reported that they did not always use condoms. In the multivariable analysis, being married $(\mathrm{OR}=1.66 ; 95 \% \mathrm{CI} 1.25-2.20)$ or cohabitating or separated, divorced, or widowed $(\mathrm{OR}=3.67 ; 1.85-7.29)$, lower educational level $(\mathrm{OR}=0.66 ; 0.46-0.94)$, greater poverty $(\mathrm{OR}=1.60 ; 1.25-2.20)$, not having HIV status disclosed $(\mathrm{OR}=0.34 ; 0.25-0.48)$, sexual partner on antiretroviral treatment $(\mathrm{OR}=0.38 ; 0.23-0.60)$, and partner alcohol use before sex $(\mathrm{OR}=1.56 ; 1.30-1.90)$ were significantly associated with inconsistent condom use in the past 3 months. The low proportion of consistent condom use among TB patients needs to be improved.
\end{abstract}

\section{Introduction}

HIV prevalence has gradually increased among newly diagnosed tuberculosis (TB) patients in South Africa, from $44 \%$ in 2006 [1] to $60 \%$ in 2010 [2]. The high rate of HIV infections among TB patients calls for urgent HIV reduction interventions in this subpopulation. A study by Zachariah et al. [3] has shown that TB patients are prone to risky sexual behaviour including unprotected sex. Since most HIV infections in South Africa are sexually transmitted [2], there is a need to encourage sexual behaviour change among TB patients as an HIV prevention measure [4]. Correct and consistent condom use is one of the effective means of HIV prevention among sexually active people. There is scarcity of information on condom use behaviour among TB patients in South Africa. Talbot et al. [5] found among tuberculosis patients in Botswana that the majority inconsistently had used a condom in their last 10 sexual encounters, among a survey of adult TB patients in Ethiopia $78 \%$ had not used condom [6], and among HIV-infected tuberculosis patients in Thailand $42 \%$ reported never using condoms at all [7]. An investigation into condom use behaviour and its associated factors is important in assisting to plan for HIV prevention programmes that focus on promoting condom use among TB patients. The aim of this study is to determine the prevalence of inconsistent condom use among TB patients and its associated factors using a sample of $4900 \mathrm{~TB}$ public primary care patients from three health districts in South Africa.

\section{Methods}

2.1. Participants and Procedures. This is a cross-sectional survey with tuberculosis patients in public primary care clinics in South Africa. Three provinces, in South Africa, with 
the highest TB caseloads were selected for inclusion in the study. One district in each province $(N=3)$ with the highest TB caseloads was ultimately included. These districts were Siyanda in Northern Cape Province, Nelson Mandela Metro in the Eastern Cape Province, and eThekwini in KwaZuluNatal Province. Within each of these three study districts, 14 public primary health care facilities were selected on the basis of the highest TB caseloads per clinic $(N=42)$. The type of health facilities were public primary health care clinic or community health centre. All new TB and new retreatment patients were consecutively interviewed within one month of antituberculosis treatment. The interview was conducted by trained external research assistants for a period of 6 months in all 42 clinics in 2011. A health care provider who identified a new TB treatment or retreatment patient (within one month on treatment) and 18 years and above informed the patient about the study and referred the patient for participation if interested. A research assistant asked for permission/consent from patients attending the public primary care facility to participate in the interview. We have received's ethical approval from the Human Sciences Research Council Research Ethics Committee (Protocol REC no.1/16/02/11). The Department of Health in South Africa has also provided approval for this study.

\subsection{Measures}

2.2.1. Socioeconomic Characteristics. A researcher-designed questionnaire was used to record information on participants' age, gender, educational level, marital status, income, employment status, dwelling characteristics, and residential status. Poverty was assessed with 5 items on the availability or nonavailability of shelter, fuel or electricity, clean water, food, and cash income in the past week. Response options ranged from 1 = "Not one day" to $4=$ "Every day of the week". Poverty was defined as higher scores on non-availability of essential items. The total score ranged from 5 to $20,5=$ being low, $6-12=$ medium, and 13-20 = high poverty. Cronbach alpha for this poverty index was 0.89 in this sample.

2.2.2. Condom Use. It was assessed with the question "How frequently did you use condoms when you had sex with your most recent sexual partner in the past three months?". Response options were 1 = always, 2 = frequently, 3 = sometimes, and $4=$ never. Inconsistent condom use was defined as not always using a condom with the most recent sexual partner in the past three months.

2.2.3. The Kessler Psychological Distress Scale (K-10). It was used to measure global psychological distress, including significant pathology which does not meet formal criteria for a psychiatric illness $[8,9]$. This scale measures the following symptoms over the preceding 30 days by asking: "In the past 30 days, how often did you feel: nervous; so nervous that nothing could calm you down; hopeless; restless or fidgety; so restless that you could not sit still; depressed; that everything was an effort; so sad that nothing could cheer you up; worthless; tired out for no good reason?". The frequency with which each of these items was experienced was recorded using a five-point likert scale ranging from "none of the time" to "all the time". This score was then summed with increasing scores reflecting an increasing degree of psychological distress. This scale serves to identify individuals who are likely to meet formal definitions for anxiety and/or depressive disorders, as well as to identify individuals with subclinical illness who may not meet formal definitions for a specific disorder [8]. This scale has been validated in HIV positive individuals in South Africa [10]. There was significant agreement between the $\mathrm{K}-10$ and the MINI-defined depressive and anxiety disorders. A receiver operating characteristic (ROC) curve analysis indicated that the K-10 showed agreeable sensitivity and specificity in detecting depression (area under the ROC curve, 0.77$)$, generalized anxiety disorder (0.78), and posttraumatic stress disorder (PTSD) (0.77) [10]. The $\mathrm{K}-10$ scale was used as a binary variable comparing scores of 30 or more or less. The internal reliability coefficient for the $\mathrm{K}-10$ in this study was alpha $=0.92$.

2.2.4. Alcohol Consumption. The 10-item Alcohol Disorder Identification Test (AUDIT) [11] assesses alcohol consumption level (3 items), symptoms of alcohol dependence (3 items), and problems associated with alcohol use (4 items). Heavy episodic drinking is defined as the consumption of six standard drinks ( $10 \mathrm{~g}$ alcohol) or more on a single occasion [11]. In South Africa, a standard drink is $12 \mathrm{~g}$ alcohol. Because AUDIT is reported to be less sensitive at identifying risk drinking in women [12], the cut-off points of binge drinking for women (4 units) were reduced by one unit as compared with men ( 5 units), as recommended by Freeborn et al. [12]. Responses to items on the AUDIT are rated on a 4-point Likert scale from 0 to 4 , for a maximum score of 40 points. Higher AUDIT scores indicate more severe levels of risk; score 8 indicates a tendency to problematic drinking including 8-19 high risk, and 20-40 probable alcohol dependence [11]. The AUDIT has been validated in HIV patients in South Africa showing excellent sensitivity and specificity in detecting MINI-defined dependence/abuse (area under the receiver-operating characteristic curve, 0.96) [13], and among TB and HIV patients in primary care in Zambia demonstrating good discriminatory ability in detecting MINI-defined current AUDs (AUDIT $=0.98$ for women and 0.75 for men) [14]. Cronbach alpha for the AUDIT in this sample was 0.92 , indicating excellent reliability.

2.2.5. Alcohol Use in the Context of Sex. Participants were asked if they and their sexual partner had used alcohol before having sex in the past three months.

2.2.6. Perceived General Health. Participants were asked-in general, would you say your health is excellent, very good, good, fair, or poor? This measure was categorized based on participant response $(1=$ very good $=$ excellent/very good, 2 = good, and 3 = fair/poor).

TB treatment status, HIV status, Sexually Transmitted Infection (STI) history, and antiretroviral treatment were assessed by self-report and from medical information. HIV testing status was assessed by self-report. 


\section{Data Analysis}

Data were analyzed using Statistical Package for the Social Sciences (SPSS) for Windows software application programme version 19.0. Frequencies, means, and standard deviations were calculated to describe the sample. Data were checked for normality distribution and outliers. For nonnormal distribution, nonparametric tests were used. Associations of inconsistent condom use, sociodemographics, health variables, and alcohol and drug use were identified using logistic regression analyses. Interaction between predictor variables was examined; none of the variables had a variance iInflation factor (VIF) value above 2.5. Following each univariate regression, multivariable regression models were constructed. Independent variables from the univariate analyses were entered into the multivariable model if significant at $P<$ 0.05 level. For each model, the $R^{2}$ are presented to describe the amount of variance explained by the multivariable model. Probability below 0.05 was regarded as statistically significant.

\section{Results}

4.1. Sample Characteristics and Inconsistent Condom Use. From the total sample $(N=4935)$ included in the study, $35(0.7 \%)$ refused to participate, so the final sample included $4900,54.5 \%$ men and $45.5 \%$ women, with a mean age of 36.2 years $(\mathrm{SD}=11.5)$, range from 18 to 93 years. Almost twothirds of the participants $(65.2 \%)$ were between 25 to 44 years old, $84.6 \%$ were Black Africans, $72.5 \%$ was never married, $27.7 \%$ had completed secondary education, and $17 \%$ scored high on the poverty index. Regarding health status of the TB patients, 55\% were coinfected with HIV, 23.3\% were retreatment $\mathrm{TB}$ patients, $46.3 \%$ rated their health status as fair or poor, and $26.2 \%$ had severe psychological distress. Twenty-two percent were on antiretroviral treatment, $63.5 \%$ had disclosed their HIV status to their last sexual partner, and $72.8 \%$ had a sexual partner with unknown or HIV negative status. Almost one in five of the participants $(23.3 \%)$ engaged in hazardous or harmful alcohol use, $11.2 \%$ had used alcohol before sex in the past three months, and 9.6\% reported that their sexual partner had used alcohol before sex in the past three months. When asked about their consistency of condom use in the past 3 months, $63.5 \%$ had used condoms inconsistently (see Table 1 ).

Table 2 shows both the bivariate analysis reporting the unadjusted odds ratios and multivariable analysis reporting the adjusted odds ratios of all possible predictors of inconsistent condom use. The bivariate analysis showed that older age, being married or cohabitating, or separated, divorced, or widowed, lower educational level, greater poverty, being HIV negative, ever having had a sexually transmitted infection (STI), severe psychological stress, not being on ART, sexual partner not on ART, not having HIV status disclosed to their last sexual partner, partner HIV status unknown or negative, alcohol use, alcohol use before sex, and partner alcohol use before sex were associated with inconsistent condom use in the past 3 months. Finally, in the multivariable analysis, being married or cohabitating or separated, divorced or widowed, lower educational level, greater poverty, not having HIV status disclosed, sexual partner on ART, and partner alcohol use before sex were significantly associated with inconsistent condom use in the past 3 months (see Table 2).

\section{Discussion}

The study found high rates of inconsistent condom use, as found in some other studies [5-7], among a large sample of TB patients in South Africa. This finding is alarming given the high rate of HIV and TB coinfection at a national level in South Africa [2]. The dual epidemics of HIV and TB have become a public health priority and is beginning to receive increasing attention from the National Department of Health as specified in the National Strategic Plan 2012-2016 [15]. $\mathrm{TB}$, therefore, cannot be managed as a single disease entity. A comprehensive treatment and prevention programme for $\mathrm{TB}, \mathrm{HIV}$, and indeed other comorbid disorders is required in order to meet this public health challenge. In the context of this study, condom use needs to be considered as one of the HIV prevention measures when planning for HIV prevention programmes for TB patients.

Further, the results of the study suggest that people who were married/cohabitating and those separated/divorced/ widowed, with lower education and living in poverty were more likely to report inconsistent condom use compared to their respective counterparts. Similar to the results suggested in this study, other studies showed that lower levels of education were associated with lack of condom use [16, 17]. Lack of condom use by married people in this study is consistent with findings from previous studies $[18,19]$. This finding may be because there is some trust built among people in such relationships leading to decisions to use condoms occasionally or stop altogether. Furthermore, it was suggested by study results that people who have not disclosed their HIV status, those whose sexual partner was not on ART, and those whose partner took alcohol before sex, were more likely to report inconsistent condom use. This finding is of concern since nondisclosure of HIV status and alcohol use in the context of sex coupled with inconsistent condom use may lead to further HIV infection [20,21]. Efforts should also be made for follow-up couple counselling and testing, use of behaviour rehearsal technique to overcome barriers of disclosure, and integrating alcohol use into HIV/AIDS risk reduction interventions.

\section{Study Limitations}

Caution should be taken when interpreting the results of this study because of certain limitations. As this was a crosssectional study, causality between the compared variables cannot be concluded. A further limitation was that most variables were assessed by self-report and desirable responses may have been given, in particular regarding condom use.

\section{Conclusion}

The control of the concomitant HIV and tuberculosis epidemics is one of the greatest challenges facing South Africa. 
TABLE 1: Sample characteristics and inconsistent condom use.

\begin{tabular}{|c|c|c|}
\hline Socioeconomic factors & $\begin{array}{c}\text { Total } \\
\mathrm{N}(\%) \text { or } \mathrm{M}(\mathrm{SD}) \\
\end{array}$ & $\begin{array}{c}\text { Inconsistent condom use } \\
\mathrm{N}(\%) \text { or } \mathrm{M}(\mathrm{SD})\end{array}$ \\
\hline All & 4900 & $2697(63.5)$ \\
\hline \multicolumn{3}{|l|}{ Age } \\
\hline $18-24$ & $643(13.3)$ & $315(57.2)$ \\
\hline $25-34$ & $1841(38.1)$ & $1004(60.4)$ \\
\hline $35-44$ & $1313(27.1)$ & $742(63.6)$ \\
\hline 45 or more & $1040(21.5)$ & $601(73.1)$ \\
\hline Male & $1631(54.5)$ & $1477(64.8)$ \\
\hline Female & $2194(45.5)$ & $1178(61.9)$ \\
\hline Never married & $3356(72.5)$ & $1772(60.8)$ \\
\hline Married/cohabitating & $1001(21.6)$ & $609(66.8)$ \\
\hline Separated/divorced/widowed & $275(5.9)$ & $165(80.9)$ \\
\hline Grade 7 or less & $1269(26.3)$ & $730(71.8)$ \\
\hline Grade 8-11 & $2213(45.9)$ & $1219(61.6)$ \\
\hline Grade 12 or more & $1336(27.7)$ & $714(59.5)$ \\
\hline \multicolumn{3}{|l|}{ Poverty index } \\
\hline Low & $1617(35.0)$ & $802(56.8)$ \\
\hline Medium & $2227(48.2)$ & $1287(64.5)$ \\
\hline High & $275(16.8)$ & $454(72.3)$ \\
\hline Black African & $4078(84.6)$ & $2277(62.9)$ \\
\hline Coloured & $634(13.1)$ & $331(66.6)$ \\
\hline Indian, Asian, White, other & $111(2.3)$ & $59(66.3)$ \\
\hline \multicolumn{3}{|l|}{ Health status } \\
\hline \multicolumn{3}{|l|}{ HIV status } \\
\hline HIV negative & $1759(36.9)$ & $954(64.1)$ \\
\hline HIV unknown & $385(8.1)$ & $258(79.6)$ \\
\hline HIV positive & $2619(55.0)$ & $1417(60.6)$ \\
\hline Ever STI & $329(7.3)$ & $212(69.1)$ \\
\hline New TB patient & $3707(76.7)$ & $2061(63.5)$ \\
\hline Retreatment TB patient & $1128(23.3)$ & $610(63.0)$ \\
\hline \multicolumn{3}{|l|}{ Perceived health status } \\
\hline Excellent/very good & $928(19.2)$ & $516(64.3)$ \\
\hline Good & $1667(34.5)$ & $916(62.7)$ \\
\hline Fair/poor & $2238(46.3)$ & $1245(63.8)$ \\
\hline Severe psychological distress & $1195(26.2)$ & $709(67.1)$ \\
\hline On ART & $906(22.0)$ & $421(53.3)$ \\
\hline Disclosed HIV status to last partner & $2769(63.5)$ & $1480(54.9)$ \\
\hline Partner with HIV unknown or negative versus positive & $3244(72.8)$ & $2010(66.6)$ \\
\hline Sexual partner on ART & $440(11.1)$ & $189(44.8)$ \\
\hline \multicolumn{3}{|l|}{ Alcohol use } \\
\hline \multicolumn{3}{|l|}{ AUDIT } \\
\hline Abstinent or low risk (score $0-7$ ) & $3688(76.7)$ & $1985(61.2)$ \\
\hline High risk (8-19) & $799(16.6)$ & $470(68.5)$ \\
\hline Probably alcohol dependent (20-40) & $321(6.7)$ & $205(76.2)$ \\
\hline Alcohol before sex in the past 3 months & $549(11.2)$ & $368(73.6)$ \\
\hline Partner alcohol before sex in the past 3 months & $408(9.6)$ & $325(75.6)$ \\
\hline
\end{tabular}


TABLE 2: Association between inconsistent condom use, socioeconomic factors, health status, and alcohol use.

\begin{tabular}{|c|c|c|}
\hline & Crude OR $(95 \% \mathrm{CI})$ & Adjusted $\mathrm{OR}^{\mathrm{a}, \mathrm{b}}(95 \% \mathrm{CI})$ \\
\hline \multicolumn{3}{|l|}{ Socioeconomic factors } \\
\hline Age & $1.02(1.02-1.03)^{* * *}$ & $1.00(0.99-1.01)$ \\
\hline Male versus female & $1.13(1.00-1.28)$ & $0.96(0.75-1.24)$ \\
\hline Never married & 1.00 & 1.00 \\
\hline Married/cohabitating & $1.30(1.11-1.52)^{* * *}$ & $1.66(1.25-2.20)^{* * *}$ \\
\hline Separated/divorced/widowed & $2.73(1.91-3.90)^{* * *}$ & $3.67(1.85-7.29)^{* * *}$ \\
\hline Grade 7 or less & 1.00 & 1.00 \\
\hline Grade 8-11 & $0.63(0.54-0.74)^{* * *}$ & $0.72(0.53-0.99)^{*}$ \\
\hline Grade 12 or more & $0.58(0.48-0.69)^{* * *}$ & $0.66(0.46-0.94)^{* *}$ \\
\hline \multicolumn{3}{|l|}{ Poverty index } \\
\hline Low & 1.00 & 1.00 \\
\hline Medium & $1.38(1.20-1.59)^{* * *}$ & $1.60(1.24-2.20)^{* * *}$ \\
\hline High & $1.98(1.62-2.43)^{* * *}$ & $1.54(1.05-2.25)^{*}$ \\
\hline Black African & 1.00 & - \\
\hline Coloured & $1.17(0.96-1.43)$ & \\
\hline Indian, Asian, White, other & $1.16(0.74-1.81)$ & \\
\hline \multicolumn{3}{|l|}{ Health status } \\
\hline HIV positive versus negative & $0.86(0.75-0.99)^{*}$ & $0.96(0.72-1.28)$ \\
\hline Ever STI & $1.38(1.07-177)^{*}$ & $1.10(0.72-1.67)$ \\
\hline New TB versus retreatment & $1.02(0.88-1.19)$ & - \\
\hline \multicolumn{3}{|l|}{ Perceived health status } \\
\hline Excellent/very good & 1.00 & - \\
\hline Good & $0.94(0.78-1.12)$ & \\
\hline Fair/poor & $0.99(0.83-1.17)$ & \\
\hline Severe psychological distress & $1.26(1.09-1.47)^{* *}$ & $1.21(0.92-1.59)$ \\
\hline On ART & $0.60(0.51-0.70)^{* * *}$ & $0.79(0.58-1.08)$ \\
\hline Disclosed HIV status to last partner & $0.32(0.28-0.37)^{* * *}$ & $0.34(0.25-0.48)^{* * *}$ \\
\hline Partner HIV unknown or negative versus positive & $1.60(1.39-1.84)^{* * *}$ & $1.18(0.87-2.12)$ \\
\hline Sexual partner on ART & $0.41(0.33-0.50)^{* * *}$ & $0.38(0.23-0.60)^{* * *}$ \\
\hline \multicolumn{3}{|l|}{ Alcohol use } \\
\hline \multicolumn{3}{|l|}{ AUDIT } \\
\hline Abstinent or Low risk $(0-7)$ & 1.00 & 1.00 \\
\hline High risk (8-19) & $1.38(1.16-1.64)^{* * *}$ & $1.14(0.82-1.58)$ \\
\hline Probably alcohol dependent (20-40) & $2.03(1.52-2.71)^{* * *}$ & $1.44(0.86-2.42)$ \\
\hline Alcohol before sex & $1.22(1.12-1.32)^{* * *}$ & $1.08(0.78-1.50)$ \\
\hline Partner alcohol before sex & $1.33(1.21-1.46)^{* * *}$ & $1.56(1.30-1.90)^{* * *}$ \\
\hline
\end{tabular}

asing "enter" LR selection of variables.

${ }^{\mathrm{b}}$ Hosmer and Lemeshow Chi-square 7.16, df 8, 0.516; Cox and Snell $R^{2} 0.15$; Nagelkerke $R^{2} 0.20$.

${ }^{*} P<0.05 ;{ }^{* *} P<0.01 ; * * * P<0.001$.

It is essential to link TB and HIV treatment and prevention programmes in situations where both diseases are prevalent to improve the diagnosis, treatment, and outcomes for patients affected by both diseases. The high rates of inconsistent condom use among TB patients found in this study needs to be improved and both male and female condom use should be considered HIV prevention measures when planning for HIV prevention programmes for TB patients.

\section{Acknowledgments}

The Department of Health in South Africa funded this study through a tender "NDOH: 21/2010-2011 Implementation and monitoring of Screening and Brief Intervention for alcohol use disorders among Tuberculosis patients" that was awarded to the HSRC.

\section{References}

[1] World Health Organization, "Global tuberculosis control. Surveillance, planning, financing,” WHO Report $2008 \mathrm{WHO} /$ HTM/TB/2008.393, World Health Organization, Geneva, Switzerland, 2008.

[2] World Health Organization, "Global tuberculosis control. Epidemiology, strategy, financing," Tech. Rep. WHO/HTM/TB/ 2009.411, World Health Organization, Geneva, Switzerland, 2011. 
[3] R. Zachariah, M. P. Spielmann, A. D. Harries, and F. L. Salaniponi, "Voluntary counselling, HIV testing and sexual behaviour among patients with tuberculosis in a rural district of Malawi," International Journal of Tuberculosis and Lung Disease, vol. 7, no. 1, pp. 65-71, 2003.

[4] G. J. C. Kigosi, P. Heunis, E. Chikobvu, E. Wouters, and H. S. van den Berg, Determinants of Condom Use Amongst Tuberculosis Patients in the Free State Province, South Africa [Ph.D. thesis], Department of Psychology, Faculty of the Humanities, University of the Free State (UFS), 2011.

[5] E. A. Talbot, T. A. Kenyon, T. L. Moeti et al., "HIV risk factors among patients with tuberculosis-Botswana 1999," International Journal of STD and AIDS, vol. 13, no. 5, pp. 311-317, 2002.

[6] T. Degefa, "Survey of protective behaviour practiced against HIV/AIDS in adult TB patients at Almata Zonal hospital," Ethiopian Medical Journal, vol. 44, no. 2, pp. 105-112, 2006.

[7] W. Mankatittham, S. Likanonsakul, U. Thawornwan et al., "Characteristics of HIV-infected tuberculosis patients in Thailand," Southeast Asian Journal of Tropical Medicine and Public Health, vol. 40, no. 1, pp. 93-103, 2009.

[8] R. C. Kessler, G. Andrews, L. J. Colpe et al., "Short screening scales to monitor population prevalences and trends in nonspecific psychological distress," Psychological Medicine, vol. 32, no. 6, pp. 959-976, 2002.

[9] R. C. Kessler, P. R. Barker, L. J. Colpe et al., "Screening for serious mental illness in the general population," Archives of General Psychiatry, vol. 60, no. 2, pp. 184-189, 2003.

[10] G. Spies, K. Kader, M. Kidd et al., "Erratum: validity of the $\mathrm{K}-10$ in detecting DSM-IV-defined depression and anxiety disorders among HIV-infected individuals," AIDS Care, vol. 22, no. 9, pp. 1163-1168, 2009.

[11] T. F. Babor and J. C. Higgins-Biddle, Brief Intervention for Hazardous and Harmful Drinking a Manual for Use in Primary Care, World Health Organziation Department of Mental Health and Substance Dependence, Geneva, Switzerland, 2001.

[12] D. K. Freeborn, M. R. Polen, J. F. Hollis, and R. A. Senft, "Screening and brief intervention for hazardous drinking in an HMO: effects on medical care utilization," Journal of Behavioral Health Services and Research, vol. 27, no. 4, pp. 446-453, 2000.

[13] L. Myer, J. Smit, L. L. Roux, S. Parker, D. J. Stein, and S. Seedat, "Common mental disorders among HIV-infected individuals in South Africa: prevalence, predictors, and validation of brief psychiatric rating scales," AIDS Patient Care and STDs, vol. 22, no. 2, pp. 147-158, 2008.

[14] N. Chishinga, E. Kinyanda, H. A. Weiss, V. Patel, H. Ayles, and S. Seedat, "Validation of brief screening tools for depressive and alcohol use disorders among TB and HIV patients in primary care in Zambia," BMC Psychiatry, vol. 11, article 75, 2011.

[15] Department of Health, South Africa, National Strategic Plan for HIV and AIDS, STIs and TB, 2012-2016, Department of Health, Pretoria, South Africa, 2011.

[16] G. Calazans, T. W. Araujo, G. Venturi, and I. França, "Factors associated with condom use among youth aged 15-24 years in Brazil in 2003," AIDS, vol. 19, supplement 4, pp. S42-S50, 2005.

[17] A. Cobat, S. Halfen, and I. Grémy, "Determinants of condom use and heterosexual multiple sexual partnership in French Antilles and French Guiana," Revue d'Epidemiologie et de Sante Publique, vol. 56, no. 3, pp. 143-157, 2008.
[18] S. Dhalla and G. Poole, "Determinants of condom use: results of the Canadian community health survey 3.1," Canadian Journal of Public Health, vol. 100, no. 4, pp. 299-303, 2009.

[19] M. Yotebieng, C. T. Halpern, E. M. H. Mitchell, and A. A. Adimora, "Correlates of condom use among sexually experienced secondary school male students in Nairobi, Kenya," Sahara Journal, vol. 6, no. 1, pp. 9-16, 2009.

[20] P. Seth, G. M. Wingood, R. J. DiClemente, and L. S. Robinson, "Alcohol use as a marker for risky sexual behaviors and biologically confirmed sexually transmitted infections among young adult African-American women," Women's Health Issues, vol. 21, no. 2, pp. 130-135, 2011.

[21] J. C. Fisher, H. Bang, and S. H. Kapiga, "The association between HIV infection and alcohol use: a systematic review and meta-analysis of African studies," Sexually Transmitted Diseases, vol. 34, no. 11, pp. 856-863, 2007. 


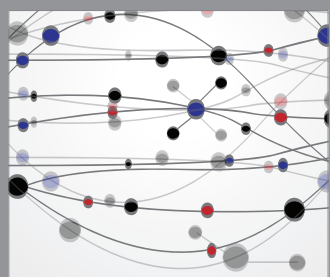

The Scientific World Journal
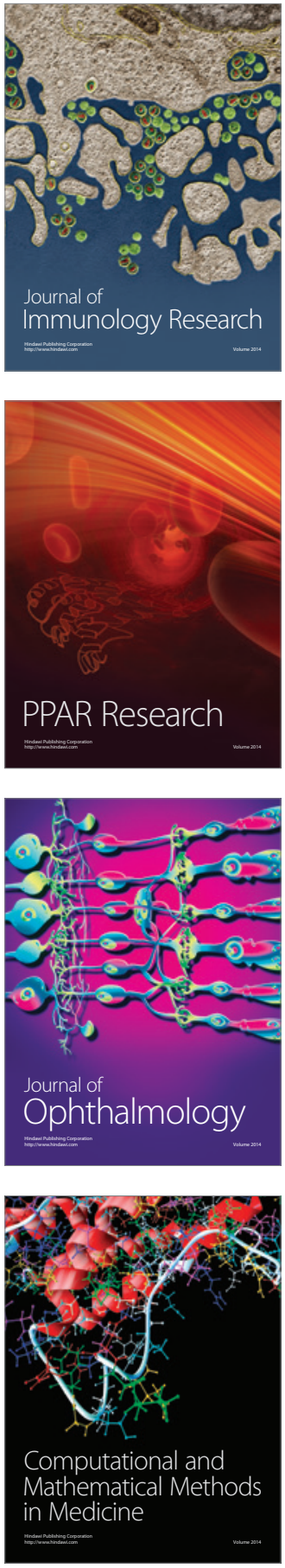

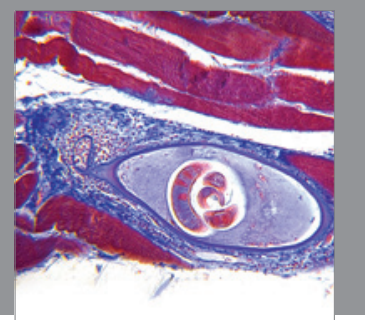

Gastroenterology

Research and Practice
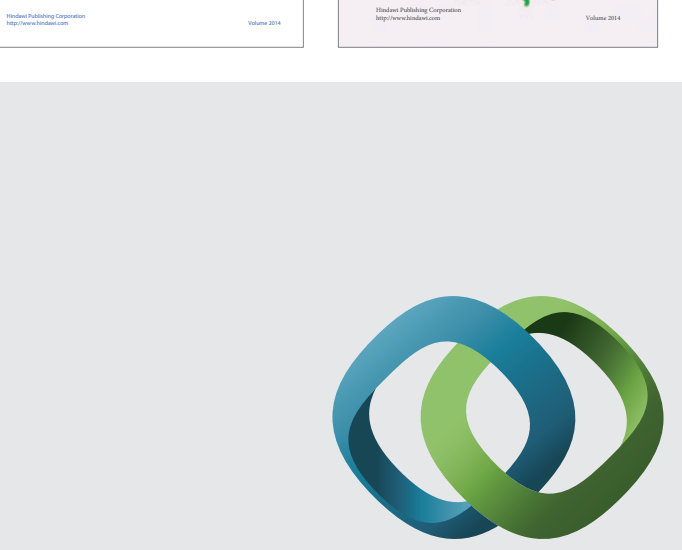

\section{Hindawi}

Submit your manuscripts at

http://www.hindawi.com
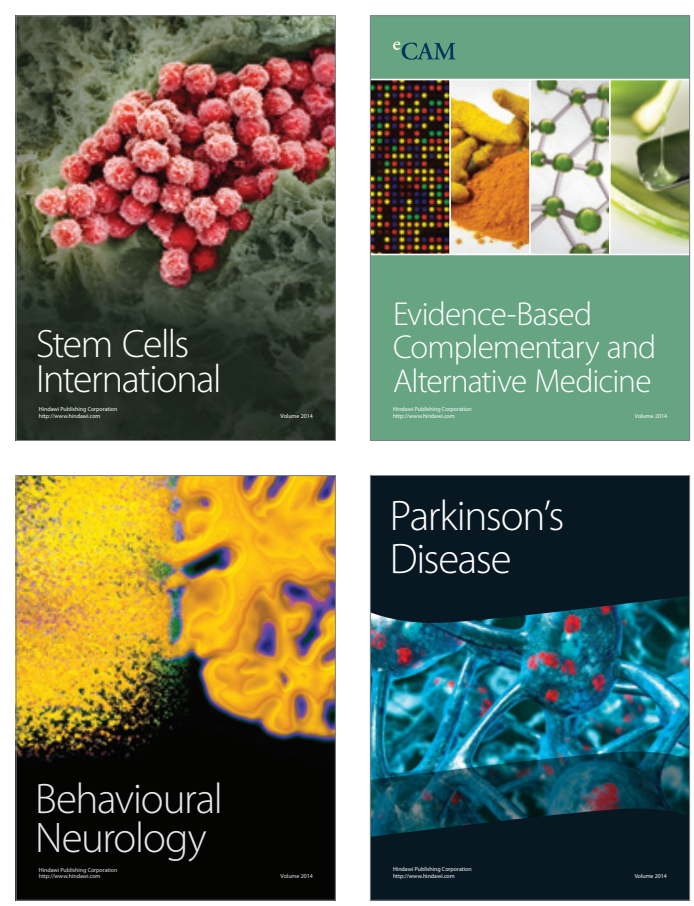

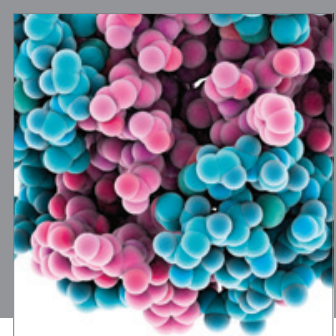

Journal of
Diabetes Research

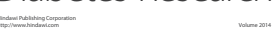

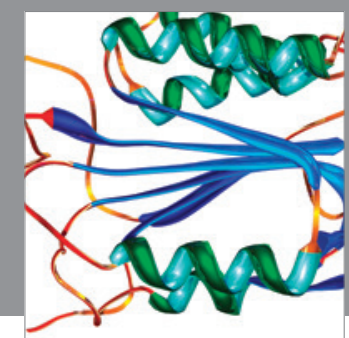

Disease Markers
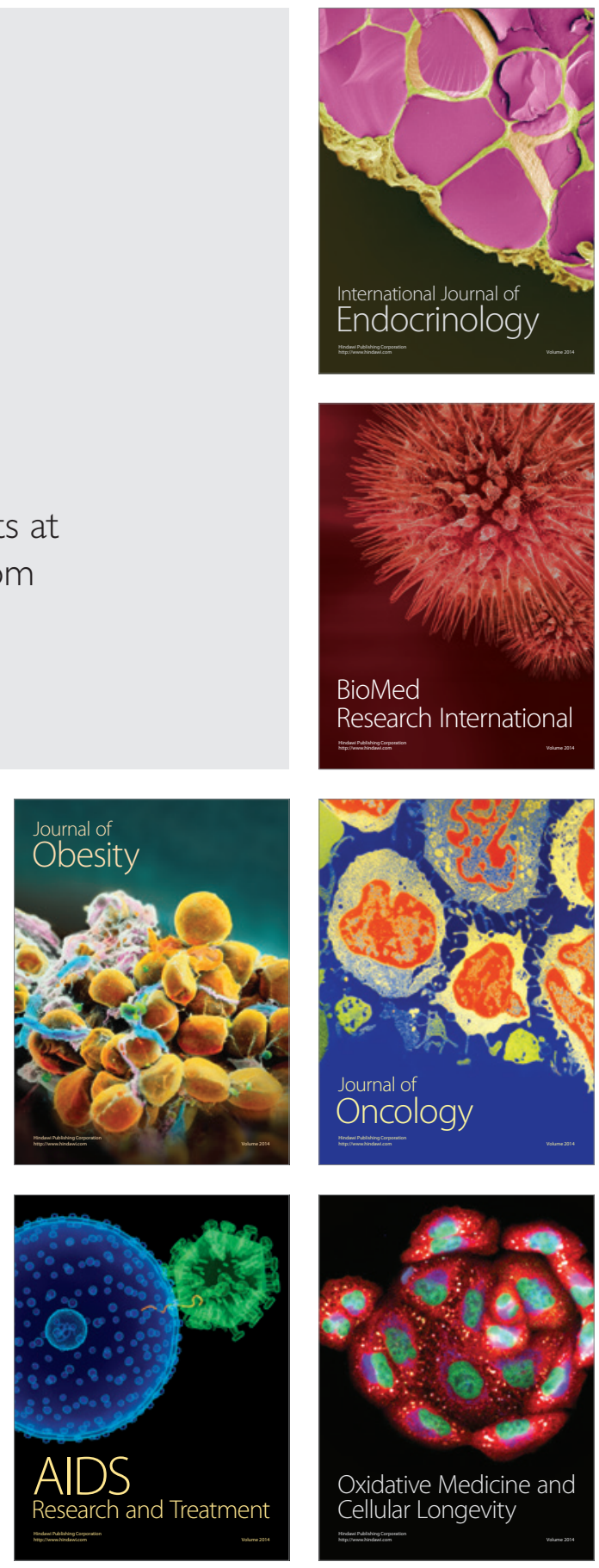